\title{
PRIMES IN ARITHMETIC PROGRESSION AND UNIFORM DISTRIBUTION
}

\author{
M. A. WODZAK
}

(Communicated by Dennis A. Hejhal)

\begin{abstract}
Let $f$ be an entire, non-polynomial function with real coefficients. Let $\tilde{p}$ run through the primes such that $\tilde{p} \equiv h \bmod k,(h, k)=1$, and let $M>1$. If there exists $\alpha \in(1,4 / 3)$ such that $|f(z)| \leq \exp \left((\log |z|)^{\alpha}\right)$ for all $|z|>M$, then the sequence $\{f(\tilde{p})\}$ is uniformly distributed modulo 1 .
\end{abstract}

In [1], R. C. Baker, improving on earlier results of Rauzy [3] and Rhin [4], demonstrated that if the function $f$ had the following properties:

Property 1. $f$ is entire,

Property 2. $f$ is non-polynomial,

Property 3. $f(\mathbb{R}) \subset \mathbb{R}$,

Property 4. There exists $\alpha=\alpha(f) \in(1,4 / 3)$, such that $|f(z)|<$ $\exp \left((\log |z|)^{\alpha}\right)$ for all complex $z$ of sufficiently large modulus,

then the sequences $\{f(n)\}$ and $\{f(p)\}$, where $n$ and $p$ run through the natural numbers and the primes respectively, are both uniformly distributed modulo 1. It is known that the 4/3 in Property 4 may not be replaced by any number greater than 2, but it is not yet known if it may be increased to any other number less than 2. The following expands on the above result of Baker in as much as it demonstrates:

Theorem. If $f$ satisfies Properties $1-4$ and $\{\tilde{p}\}$ is the sequence of primes such that $\tilde{p} \equiv h \bmod k,(h, k)=1$, then the sequence $\{f(\tilde{p})\}$ is uniformly distributed modulo 1 .

Before proving the theorem, we need the following:

Lemma. If $f$ satisfies Properties $1-4$, then so does $f_{\theta}$ where

$$
f_{\theta}(z)=f(z)+\theta z, \quad \theta \in(-1,1) .
$$

That this function satisfies Properties $1-3$ is obvious, and its satisfaction of Property 4 is shown by simple calculation. (Of course, the distribution modulo 1 of $\left\{f_{x}\left(a_{j}\right)\right\}, a_{j} \in \mathbb{Z}$, is exactly that of $\left\{f_{x-[x]}\left(a_{j}\right)\right\}$ for any real $\left.x.\right)$

Received by the editors April 14, 1993.

1991 Mathematics Subject Classification. Primary 11K06, 11L20, 11 N13.

This paper will constitute part of the author's Ph.D. thesis at the University of MissouriColumbia. 
It is apparent in the proof of the theorem that if the $4 / 3$ in Baker's theorem should, at any point in the future, be increased to some other number less than 2 , then the uniform distribution of $\{f(\tilde{p})\}$ would automatically follow.

Throughout the remainder of this note, we will use the notation $a \equiv_{c} b$ to denote $a \equiv b \bmod c$ and $e(x)$ to denote $\exp (2 \pi i x)$.

Proof of Theorem. Demonstrating that $\{f(\tilde{p})\}_{\tilde{p} \equiv \sum_{k} h}$ is uniformly distributed modulo 1 is, by Weyl's Criterion, the equivalent of showing

$$
\lim _{N \rightarrow \infty} \frac{\log N}{N} \sum_{\substack{\tilde{p} \leq N \\ \tilde{p} \equiv_{k} h}} e(l f(\tilde{p}))=0, \quad \text { for each } l \in \mathbb{N} .
$$

To this end we note that

$$
S:=\left|\sum_{\substack{\tilde{p} \leq N \\ \tilde{p} \leqq \equiv_{k} h}} e(l f(\tilde{p}))\right|=\left|\sum_{p \leq N} e(l f(p)) \frac{1}{\varphi(k)} \sum_{j=1}^{\varphi(k)} \chi_{j}(p) \overline{\chi_{j}}(h)\right|
$$

where the $\chi$ are the Dirichlet Characters $\bmod k$.

Clearly, there is some particular character $\chi$ such that

$$
\begin{aligned}
S & \leq \mid \sum_{p \leq N} e(l(f(p)) \chi(p) \mid \\
& = \begin{cases}\left|\sum_{p \leq N,(p, k)=1} e(l f(p))\right| & \chi \text { principal, } \\
\left|\sum_{p \leq N,(p, k)=1} e(l f(p)) \frac{\tau_{k_{1}}}{k_{1}} \sum_{a=1}^{k_{1}} \bar{\chi}_{1}(a) e\left(-\frac{a}{k_{1}} p\right)\right| & \text { otherwise, }\end{cases}
\end{aligned}
$$

where $k_{1} \in(1, k]$ divides $k$ and $\chi$ is induced by $\chi_{1} \bmod k_{1}$. It is well known (see, for example, [2], Theorem 7.4.4) that the Gauss sum

$$
\tau_{k_{1}}=\sum_{m=1}^{k_{1}} \chi_{1}(m) e\left(m / k_{1}\right)
$$

has modulus $\sqrt{k_{1}}$. Thus,

$$
\begin{aligned}
S & \leq \begin{cases}\left|\sum_{p \leq N} e(l f(p))\right|+k & \chi \text { principal }, \\
\left|\sum_{p \leq N} e(l f(p)) \frac{\tau_{k_{1}}}{k_{1}} \sum_{a=1}^{k_{1}} \bar{\chi}_{1}(a) e\left(-\frac{a}{k_{1}} p\right)\right|+k^{3 / 2} & \text { otherwise, }\end{cases} \\
& \leq k^{1 / 2}\left|\sum_{p \leq N} e\left(l f(p)-\frac{b}{k} p\right)\right|+k^{3 / 2} \quad \text { for some } b=1, \ldots, k
\end{aligned}
$$

And so

$$
\lim _{N \rightarrow \infty} \frac{\log N}{N} S \leq k^{1 / 2} \lim _{N \rightarrow \infty} \frac{\log N}{N}\left|\sum_{p \leq N} e\left(l\left(f_{-\frac{k}{k l}}(p)\right)\right)\right|+k^{3 / 2} \lim _{N \rightarrow \infty} \frac{\log N}{N} .
$$

Now, by the lemma and Baker's theorem, $\left\{f_{-b / k l}(p)\right\}$ is uniformly distributed mod 1 for all $-b / k l$, which is equivalent to the statement that

$$
\lim _{N \rightarrow \infty} \frac{\log N}{N} \sum_{p \leq N} e\left(n\left(f_{-\frac{k}{k l}}(p)\right)=0 \text { for each } n \in \mathbb{N} .\right.
$$


In particular, the limit vanishes when $n=l$.

Remark. In the much simpler though related case of indexing with an arithmetic sequence in its entirety, it is apparent that $f_{h, k}$ defined by

$$
f_{h, k}(z)=f(k z+h), \quad h, k \in \mathbb{N},
$$

satisfies Properties 1-4 if $f$ does, and so, under those conditions, $\{f(n)\}_{n \equiv_{k} h}=$ $\{f(m k+h)\}$ is also uniformly distributed mod 1 . Moreover, the reader may easily verify that under the same hypotheses, yet more uniformly distributed sequences will be produced if $f$ is indexed with such sequences as $\left\{n^{m}\right\}$ and $\left\{p^{m}\right\}$ where $m \in \mathbb{N}$ is constant.

\section{REFERENCES}

1. R. C. Baker, Entire functions and uniform distribution modulo 1, Proc. London Math. Soc. (3) 49 (1984), 87-110.

2. Hua Loo Keng, Introduction to number theory, Springer, New York, 1982.

3. G. Rauzy, Fonctions entières et répartition modulo un, II, Bull. Soc. Math. France 101 (1973), 185-192.

4. G. Rhin, Répartition modulo 1 de $f\left(p_{n}\right)$ quand $f$ est une série entière, Répartition Modulo 1, Lecture Notes in Math., vol. 475, Springer, Berlin, 1975, pp. 176-244.

Department of Mathematics, University of Missouri, Columbia, Missouri 65211

E-mail address: mathgr40 mizzou1 